ARTICLE OPEN

\title{
Asymmetric photocurrent extraction in semitransparent laminated flexible organic solar cells
}

\author{
Jonas Bergqvist ${ }^{1}$, Thomas Österberg ${ }^{1}$, Armantas Melianas ${ }^{1}$, Luis Ever Aguirre ${ }^{1}$, Zheng Tang ${ }^{2}$, Wanzhu Cai ${ }^{1}$, Zaifei Ma $^{2}$, \\ Martijn Kemerink $\mathbb{B D}^{3}$, Desta Gedefaw ${ }^{4}$, Mats R. Andersson ${ }^{4,5}$ and Olle Inganäs ${ }^{1}$
}

Scalable production methods and low-cost materials with low embodied energy are key to success for organic solar cells. PEDOT (PSS) electrodes meet these criteria and allow for low-cost and all solution-processed solar cells. However, such devices are prone to shunting. In this work we introduce a roll-to-roll lamination method to construct semitransparent solar cells with a PEDOT(PSS) anode and an polyethyleneimine (PEI) modified PEDOT(PSS) cathode. We use the polymer:PCBM active layer coated on the electrodes as the lamination adhesive. Our lamination method efficiently eliminates any shunting. Extended exposure to ambient degrades the laminated devices, which manifests in a significantly reduced photocurrent extraction when the device is illuminated through the anode, despite the fact that the PEDOT(PSS) electrodes are optically equivalent. We show that degradation-induced electron traps lead to increased trap-assisted recombination at the anode side of the device. By limiting the exposure time to ambient during production, degradation is significantly reduced. We show that lamination using the active layer as the adhesive can result in device performance equal to that of conventional sequential coating.

npj Flexible Electronics (2018)2:4 ; doi:10.1038/s41528-017-0017-6

\section{INTRODUCTION}

There is an urgent need to replace the present fossil-based energy production with sustainable energy sources. Thin-film solar cells and organic photovoltaics (OPV) in particular are technologies with a large potential to contribute to the energy transition due to very short energy payback times ${ }^{1}$ and scalable production methods. ${ }^{2}$ The best devices approach $12 \%$ power conversion efficiency $(P C E)^{3}$ and due to improved OPV performance under low light-intensity conditions the yearly-average energy output is improved, reducing the competitive gap between OPV and silicon photovoltaics. Nevertheless, the OPV technology is not intended to compete with the high PCE of silicon-based solar cell installations but is instead targeted for entry markets that require the unique features of OPV, such as curved form factors enabled by flexible substrates, color tunability, semitransparency, low weight and the uniquely low energy payback times.

To realize these advantages materials and production methods must be scalable with high throughput, such as roll-to-roll printing under ambient atmosphere. Vacuum-based processing, commonly used to deposit reflective (such as aluminum/chromium or silver) or transparent metal electrodes (indium tin oxide (ITO)), should ideally be avoided due to their high energy input. ${ }^{4}$ Indium in ITO is in addition too scarce to be a scalable alternative. Solution-processed electrodes have a significantly lower embodied energy and allow for the high speed printing needed for scalable production. To fully utilize the benefits of solution processing all layers in the device should be coated or printed.

The use of semitransparent Poly(3,4-ethylenedioxythiophene)poly(styrenesulfonate), PEDOT(PSS) electrodes for both the anode and the cathode allows for the rapid printing of OPV modules at a small energy budget and a potentially short energy payback time. Highly conductive PEDOT(PSS) dispersions have been proven to be a sustainable substitute for ITO ${ }^{5-7}$ as the transparent electrode. The high work function of PEDOT(PSS) makes it a suitable anode without further modification. The work function of PEDOT(PSS) can be modified, converting it to a cathode electrode, e.g., by a layer of for example $\mathrm{ZnO}^{8}$ or a thin layer of polyethyleneimine $(\mathrm{PEI})^{9}$ on top of PEDOT(PSS). Solution processable, flexible and semitransparent PEDOT(PSS) electrodes are thus a promising candidate for the upscaling and industrialization of OPV.

However, solution-cast solar cells with both electrodes being PEDOT(PSS) typically suffer from a low manufacturing yield, mainly due to a high probability of short-circuiting (or shunting), ${ }^{10}$ an issue we have experienced in the past. ${ }^{11,12}$ The shunting problem arises following the wet deposition of the top PEDOT(PSS) electrode and is avoided when the top electrode is evaporated. The actual mechanism leading to this shunting behavior is presently under discussion. 8,10 By applying a high reverse bias pulse the shunts can be eliminated and the device in most cases starts functioning. Shunting may be caused by the contact between the top and the bottom electrodes, ${ }^{10}$ or due to a nonselective PEDOT(PSS) anode. The bias pulse is suggested to electrochemically de-dope the PEDOT(PSS) anode to switch it to an electron blocking layer. ${ }^{8}$ The exact mechanism for the shunting behavior remains unclear. Regardless of the origin, shunting adds a possibly rate-limiting step to the manufacturing process ${ }^{13}$ and limits the geometric fill factor (FF) for metal-free modules, as all

\footnotetext{
${ }^{1}$ Biomolecular and Organic Electronics, IFM, Linköping University, Linkoping SE-58183, Sweden; ${ }^{2}$ Dresden Integrated Center for Applied Physics and Photonic Materials (IAPP) and Institute for Applied Physics, Technische Universität Dresden, Nöthnitzer Str. 61, Dresden 01187, Germany; ${ }^{3}$ Complex Materials and Devices, IFM, Linköping University, Linkoping

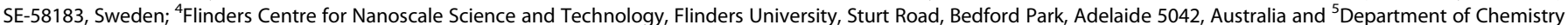
and Chemical Engineering, Polymer Technology, Chalmers, University of Technology, Goteborg SE-412 96, Sweden

Correspondence: Olle Inganäs (oling@ifm.liu.se)
}

Received: 14 May 2017 Revised: 26 October 2017 Accepted: 4 November 2017

Published online: 05 February 2018 


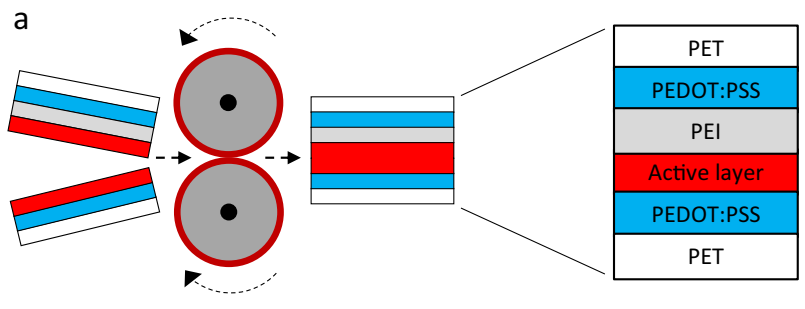

b

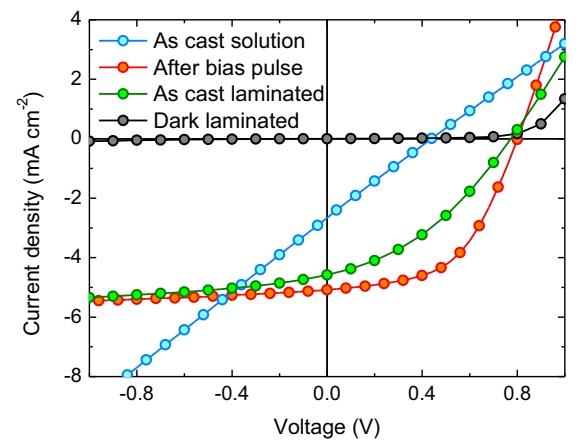

Fig. 1 Laminated solar cells a Schematic of roll-to-roll lamination using the active layer as the adhesive. b IV curves for laminated (green), laminated dark current (gray) and sequentially solution-cast TQ1:PC ${ }_{71} B M$ solar cells as cast (blue) and following a reverse bias pulse (red)

subcells must be accessible for the application of the electric pulse.

Lamination, in this respect, can be a useful method to avoid short-circuits, as it avoids the wet deposition of the top electrode. Lamination of electronic devices based on soft organic materials was demonstrated with the development of poly(3-alkylthiophenes) around $1987 .{ }^{14}$ Lamination of PEDOT(PSS) electrodes on top of the active layer using adhesive additives has also been used to construct OPV devices. ${ }^{15-19}$ Active layer lamination has previously been demonstrated for polymer/polymer blends ${ }^{20}$ and also polymer/PCBM bilayers, ${ }^{21}$ but with poor PCE below $0.1 \%$.

In this work we show that the polymer:PCBM bulk heterojunction (BHJ), coated on flexible PEDOT(PSS) electrodes, works as an excellent lamination adhesive to generate well performing solar cells, removing the need for adhesive additives. We laminate an active layer (AL1) coated on a semitransparent anode to another active layer (AL2) coated on a semitransparent cathode using a scalable roll lamination method. By lamination we obtain all solution-processed solar cells without any shunts. The laminated devices are unaffected by electric pulses, which shows that non-shunted solar cells with PEDOT(PSS) as both electrodes can be produced if wet deposition of the top electrode is avoided. We have laminated a number of different polymer: PCBM material systems to show the generality of the lamination method, mainly focusing on blends of the polymer poly(2,3-bis-(3octyloxyphenyl) quinoxaline-5,8-diyl-alt-thiophene-2,5-diyl) $(\mathrm{TQ} 1)^{22}$ with $\mathrm{PC} \mathrm{C}_{71} \mathrm{BM}$, enabling PCE of $6 \%$ in devices with an evaporated reflective electrode. TQ1 has also been our first choice when upscaling synthesis and deposition methods for roll-to-roll printing methods.

Unfortunately, ambient processing causes degradation of laminated devices, severely limiting their performance. This degradation manifests itself as a surprising asymmetry in the extracted photocurrent, depending on whether the device is illuminated through the anode or the cathode electrode, with a higher current extracted by illuminating through the cathode. We locate a zone in the $\mathrm{BHJ}$ close to the anode with reduced photo current extraction due to a high electron trap density leading to trap-assisted recombination in the active layer. We show that by reducing the time of ambient exposure we can reduce photocurrent asymmetry, i.e., degradation, and thereby attain all solution-processable PEDOT(PSS) solar cells with a performance that is comparable to sequentially cast devices.

\section{RESULTS AND DISCUSSION}

The PEDOT(PSS) electrodes are coated in ambient on polyethylene terephthalate (PET) substrates using a roll-to-roll slot-die coater. AL1 is spin-die or slot-die (bench top) coated on top of the anode stack PET/PEDOT(PSS) and AL2 on the cathode stack PET/PEDOT (PSS)/PEI. In Fig. 1a the roll lamination method using flexible substrates with the active layer as the adhesive is illustrated. Here, we demonstrate the method using two identically processed $\mathrm{BHJ}$ active layers composed of $\mathrm{TQ} 1: \mathrm{PC}_{71} \mathrm{BM}$ laminated with a roll-to-roll laminator at $120^{\circ} \mathrm{C}$ in ambient atmosphere.

Typical dark and 1 sun illumination current density vs. voltage (JV) curves from a laminated $\mathrm{TQ1}: \mathrm{PC}_{71} \mathrm{BM}$ based solar cell are shown in Fig. 1b together with an all-solution cast TQ1:PC $C_{71} B M$ device before and after a high reverse bias pulse to remove the shunting behavior. ${ }^{11}$ The active layers of both devices were spincoated in a nitrogen glove box. The performance of the laminated device shows identical $V_{\mathrm{OC}}$ but a slightly lower $J_{\mathrm{SC}}$ and FF compared to the sequentially solution-cast device following an electrical pulse. The dark current of the laminated device confirms that the problems with shunted devices in solution deposited PEDOT(PSS) based solar cells are completely avoided using active layer lamination. The laminated device is also not affected by subsequent reverse bias electrical pulses (not shown). Lamination thus allows the construction of non-shunted solar cells with PEDOT(PSS) as both the top and the bottom electrode. Since the solar cell JV curves are unaffected by electrical pulses, we conclude that shunting is not intrinsic to PEDOT(PSS), but is instead due to the deposition method.

Lamination sets the adhesive layer thicknesses to between 50 and $200 \mathrm{~nm}$. Thus, this active layer lamination method is sensitive to dust particles, which are commonly in the micrometer size range. Nevertheless, laminated devices, prepared in our regular lab, i.e., not in a clean room, are functioning with satisfactory mechanical adhesion and good electrical contact. To address the details of lamination homogeneity photoluminescence (PL), optical microscopy and photocurrent imaging have been used, shown in the supporting information Supplementary Fig. SI1, 2.

Unfortunately, ambient processing causes degradation, manifested as an asymmetry in the extracted photocurrent, with a higher photocurrent extracted by illuminating the solar cell through the cathode side than through the anode side. Below we investigate the origin of this degradation to show that it can be avoided by limiting the exposure time to ambient.

In the laminated semitransparent devices the extracted photocurrent differs significantly depending on the side of illumination. The JV curves show a $\sim 45 \%$ higher photocurrent when the laminated cell processed in ambient conditions is illuminated from the cathode side (Fig. 2). Investigations of optical transmission, reflection and absorption have clarified that parasitic absorption in the electrodes is not the cause of this large asymmetry in the extracted photocurrent (Supplementary Fig. SI3). The presence of a thin $\approx 5-10 \mathrm{~nm}$ layer of PEI on the cathode only weakly affects the optical power dissipated in the device stack, and cannot account for the large variations observed. We have also confirmed these observations by calculating the optical power dissipation in the active layer with the transfer matrix method (TMM) ${ }^{23}$ that shows a photocurrent difference of less than some few percent depending on what electrode is illuminated (Supplementary Fig. SI4), irrespective of the active layer thickness. We also discard BHJ morphology variations induced by variations in surface energy of the PEDOT(PSS) and PEDOT(PSS)/PEI surfaces 
from PL measurements. Both AL1 and AL2 are spectrally identical and also comparable in PL quenching efficiency, both for films prior to lamination and for the device excited through the anode and the cathode after lamination (Supplementary Fig. SI5). The identical PL spectra and PL quenching indicate that charge generation should be comparable in AL1 and AL2. Thus, we conclude that neither optical in-coupling nor morphologyinduced charge carrier generation differences are the main cause for the asymmetric photocurrent extraction.

To eliminate the influence of ambient conditions, it would be ideal to fully prepare the laminated devices in a controlled atmosphere, e.g., a glovebox filled with nitrogen. However, our current lamination tools are not suitable to do this. Instead, we have deposited the active layers under inert conditions and then immediately laminated them in ambient. We observe that the photoelectrical asymmetry is suppressed when the exposure to ambient is reduced. Figure 3 shows JV-curves from laminated TQ1: $\mathrm{PC}_{71} \mathrm{BM}$ solar cells with the ALs spin-coated inert and then exposed to 60, 15 and 2 min of ambient atmosphere prior to lamination. The evolution of the asymmetry is clearly visible. Longtime exposure results in a reduction in the anode photocurrent over the full measured voltage range, whereas short exposure to ambient for 15 min leads to a pronounced s-kink in the JV curve. Short ambient exposure time ( $2 \mathrm{~min})$ eliminates the photocurrent asymmetry almost completely. Ambient exposure does not only degrade the photocurrent extraction upon illumination
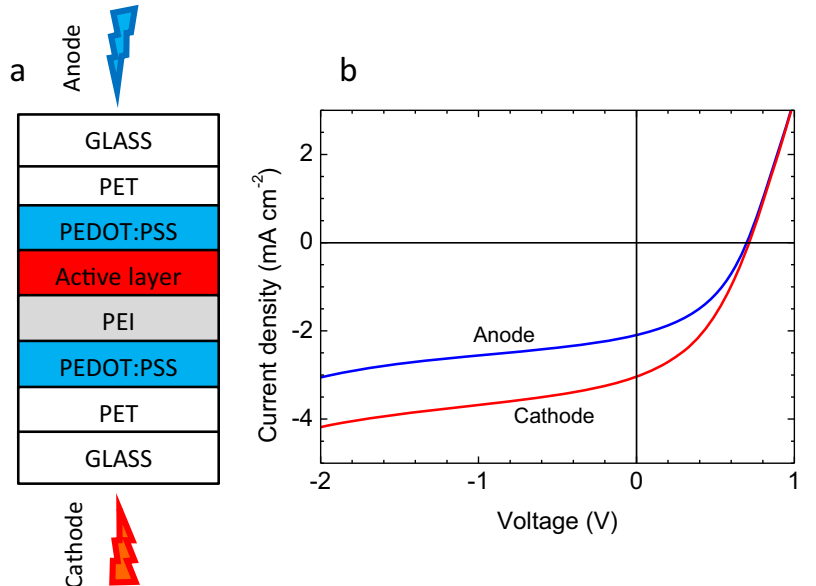

Fig. 2 Assymetric photo current a Schematic of the laminated solar cell stack illuminated either through the anode (blue) or the cathode (red) side. b JV curves for an ambient-processed solar cell illuminated through the anode (blue) and through the cathode (red) through the anode, but also for illumination through the cathode. Sixty minutes of ambient exposure reduces $J_{\mathrm{SC}}$ by over $20 \%$ (Fig. 3c). Therefore, exposure to ambient conditions during production should be prevented to avoid degradation when producing organic solar cells by lamination using the $\mathrm{AL}$ as the adhesive.

Above we have shown that ambient processing and inert processing of the active layer with subsequent exposure to ambient conditions degrades the device. The loss of photocurrent is largest upon illumination through the anode. To avoid losses the active layer must be cast under inert conditions. However, inert processing of the active layer is not fully compatible with scalable and cost-efficient production methods needed for large area OPV. Hence, a deeper understanding of the cause of asymmetric photocurrent generation is required. Since we have already ruled out optical effects and problems with charge generation, the cause must be related to the transport and/or extraction of charge carriers.

Measurements of the external quantum efficiency (EQE) as function of wavelength show significant differences when the laminated cells are illuminated from different sides (Fig. 4a). As we have shown above, the difference in photocurrent generation and hence EQE cannot be understood from a purely optical point of view. A comparison of the absorption spectrum of the pure $A L$ to the EQE spectrum indicates that carriers generated close to the anode do not contribute to the extracted photocurrent to the same degree as those generated close to the cathode (Fig. 4a). Upon illumination through the cathode, the EQE spectrum follows the absorption spectrum of the active material, while this is not the case for illumination through the anode (Fig. 4a). To understand these effects we have performed optical TMM simulations where a fraction of the active layer is assumed to have a considerably lower internal quantum efficiency (IQE).

Figure $4 \mathrm{~b}$ shows a TMM simulated EQE spectrum of an active layer which has a dead zone from where no photocurrent is extracted $(0 \% \mathrm{IQE})$, corresponding to $1 / 3$ of the total $A L$ thickness, stretching from the anode interface and into the device (Fig. 4c). The simulation is in good agreement with experiment (Fig. 4b). To probe the sensitivity of our simulations to different scenarios we have also extended the $0 \%$ IQE layer from the anode all the way to the lamination interface, corresponding to half of the laminated cell, i.e., the entire thickness of AL1. Assuming 75\% IQE in AL2 results in an EQE spectrum that also gives an adequate fit to the experimental data. Thus, we conclude that charge carriers generated in an area stretching from the anode and possibly through the full thickness of AL 1 cannot at all or can only partially be extracted.
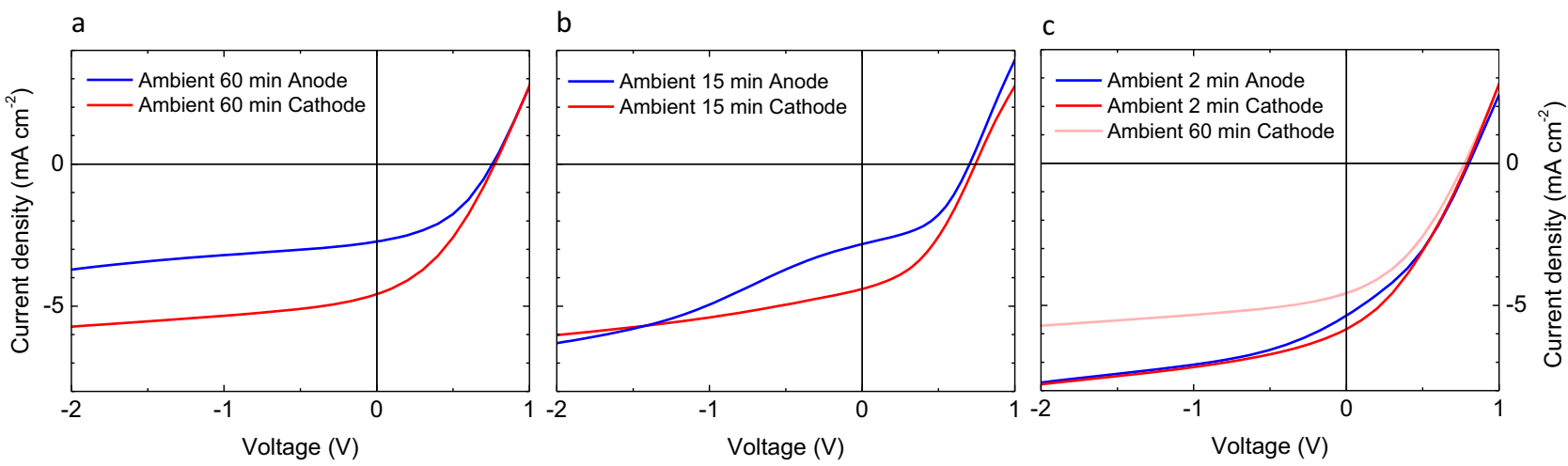

Fig. 3 Ambient exposure JV curves of laminated TQ1:PC ${ }_{71} \mathrm{BM}$ solar cells (illuminated under one sun conditions) deposited under inert conditions and exposed to ambient for a $60, \mathbf{b} 15$ and c 2 min 

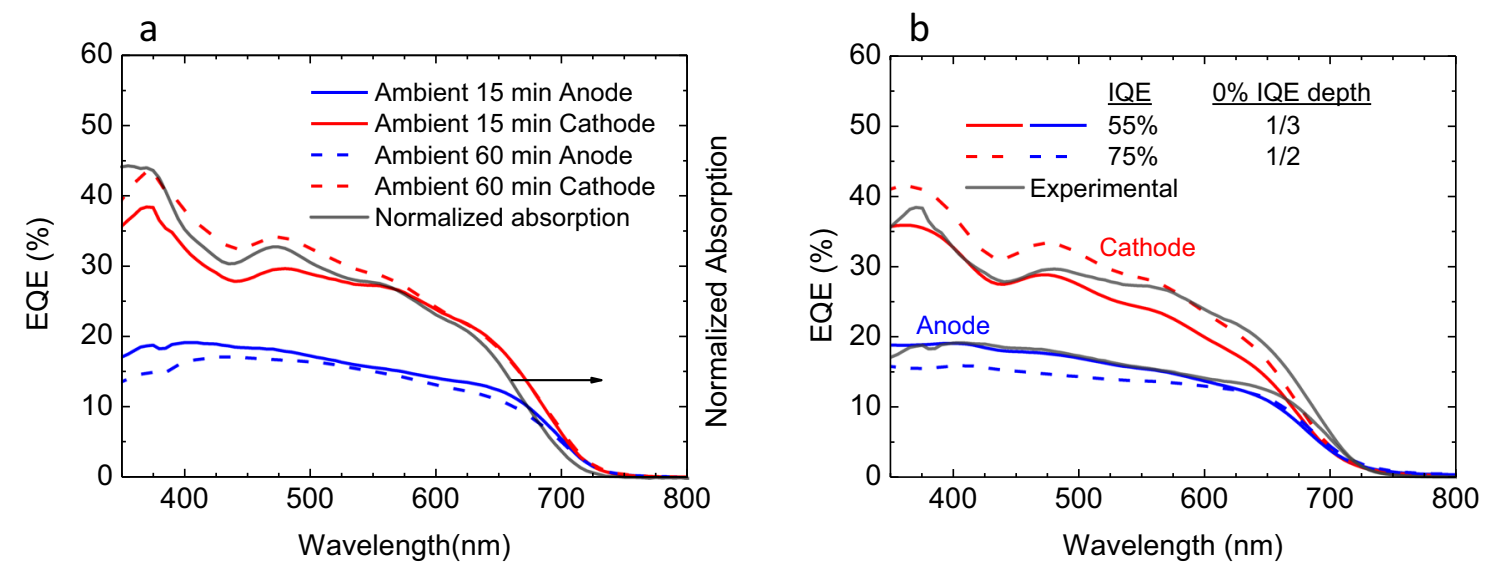

$$
\text { C }
$$

AL2
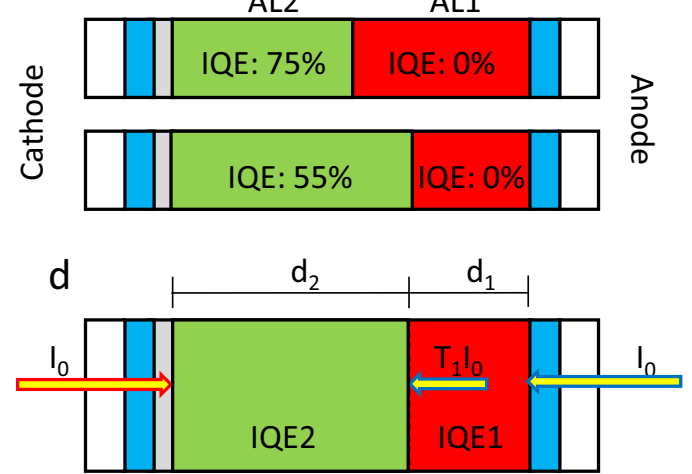

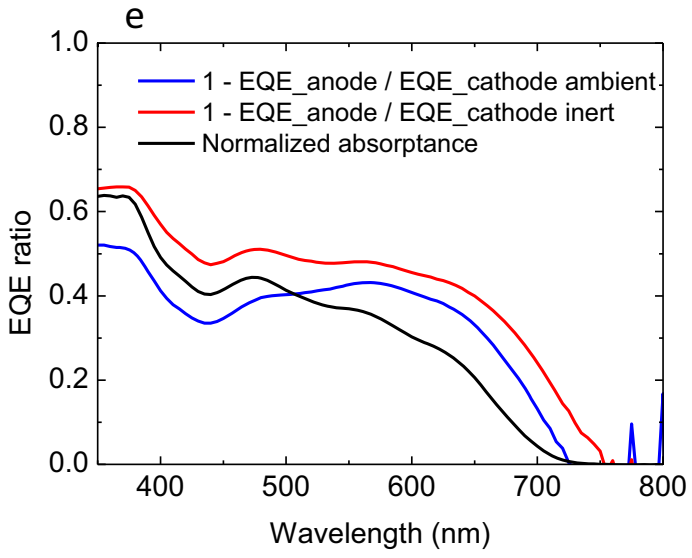

Fig. 4 Non contributing zone a Experimental EQE for devices exposed to ambient for 60 (dash) and 15 (solid) minutes illuminated through the anode (blue) and cathode (red), and the active layer absorption (black). $\mathbf{b}$ TMM simulated EQE for devices with $0 \%$ IQE on the anode half and $55 \%$ IQE on the cathode half (solid), $0 \%$ IQE in $1 / 3$ of the device close to the anode and $75 \%$ IQE in the cathode side (dash) and experimental EQE from panel a (gray). c Illustration of the position of the areas with different IQE used in $\mathbf{b}$. The active layer is divided in working (green) and dead (red) areas. $\mathbf{d}$ Schematic illustration of the (1-EQE ratio) calculation. A part of the active layer with thickness $d_{1}$ and $0 \%$ IQE on the anode side reduces the illumination intensity incident upon the working part by transmission through the dead layer, $\mathrm{T}_{1}$. e $1-\mathrm{EQE}$ ratio for a device cast under inert conditions (red), a device exposed to ambient (blue) and the scaled absorption coefficient of the AL (black)

We further verify the presence of an area with $I Q E=0 \%$ in $A L 1$ by plotting the quantity $\left(1-\mathrm{EQE}_{\text {Anode }} / \mathrm{EQE}_{\text {Cathode }}\right)$ together with the normalized absorptance of the active layer (Fig. 4e). This ratio is comparable to the absorbance of AL1, as can also be shown analytically assuming $\mathrm{IQE}=0 \%$ in $\mathrm{AL} 1$,

$$
1-\frac{\mathrm{EQE}_{\text {Anode }}}{\mathrm{EQE}_{\text {Cathode }}}=1-\frac{A_{\mathrm{A}, 2} \mathrm{IQE}_{2}+A_{\mathrm{A}, 1} \mathrm{IQE}_{1}}{A_{\mathrm{C}, 1} \mathrm{IQE} \mathrm{Q}_{1}+A_{\mathrm{C}, 2} \mathrm{IQE}_{2}}
$$

$1-\frac{A_{\mathrm{A}, 2} \mathrm{IQE}_{2}}{A_{\mathrm{C}, 2} \mathrm{IQE}}=1-\frac{A_{\mathrm{A}, 2}}{A_{\mathrm{C}, 2}}=1-\frac{A_{2} T_{1} l_{0}}{A_{2} I_{0}}=1-T_{1}=A_{1}=\exp \left(-a d_{1}\right)$

with $\mathrm{A}_{\mathrm{x}, \mathrm{i}}=A_{\mathrm{i}} \mathrm{l}_{\mathrm{x}}$ where $\mathrm{A}_{\mathrm{x}, \mathrm{i}}$ is the absorption in layer $i$ by illuminating through side $X, A_{\mathrm{i}}$ the absorptance of layer $i$ and $I_{X}$ the light intensity incident on layer $i$. The two areas and the light paths are shown in Fig. 4d. Here, we assume equal light intensity incident from both sides and internal quantum efficiency equal to $\mathrm{IQE}_{2}$ in the contributing part of the device, irrespective of illumination side. The absorption of the active layer resembling (1 $\left.-\mathrm{EQE}_{\text {Anode }} / \mathrm{EQE}_{\text {Cathode }}\right)$ implies that when illuminating from the anode side the generated charge carriers close to the anode are not collected, thus this part of the device acts as an optical filter where light is absorbed but does allow for the extraction of photocurrent. Hence, from EQE measurements and optical simulations we conclude that the non-contributing zone is localized in AL1, stretching from the anode towards the lamination interface.
To investigate what causes the asymmetry, we use electrical measurements. The lower charge collection efficiency upon illumination from the anode suggests that for ambient-exposed devices the electron transport may be significantly less efficient than the hole transport. This is due to the fact that upon illumination from the anode side, more electrons (and holes) are photo-generated at the anode side, i.e., far away from the cathode, making it more difficult to reach the cathode electrode without recombining. We have studied electron-only and holeonly devices with the active layer immediately laminated and also exposed to ambient. This can be done in a very similar structure as the transparent PEDOT(PSS) electrodes can be used as selective electrodes for hole-only devices, and PEDOT(PSS)/PEI for electrononly devices. Space-charge-limited current (SCLC) measurements indicate that exposure to ambient has a dramatic effect on electron transport (Fig. 5a). The hole mobility of a laminated TQ1: $\mathrm{PC}_{71} \mathrm{BM} 1: 3 \mathrm{BHJ}$ is unaffected by ambient exposure, while electron transport deteriorates significantly. The devices behave identical both under forward and reverse bias, shown in Supplementary Fig. SI6. The JV curve of the electron-only device behaves drastically different after ambient exposure. The characteristic shape of the degraded electron only JV curve $\left(J \propto V^{6}\right)$ is as expected from a device with a very high trap density, as previously described by e.g., Blom et al. ${ }^{24}$ Figure $5 a$ thus implies the presence of a significant electron trap density after exposure to ambient.

The presence of electron traps may cause a loss of charge carriers to trap-assisted recombination. Blom et al. have shown 

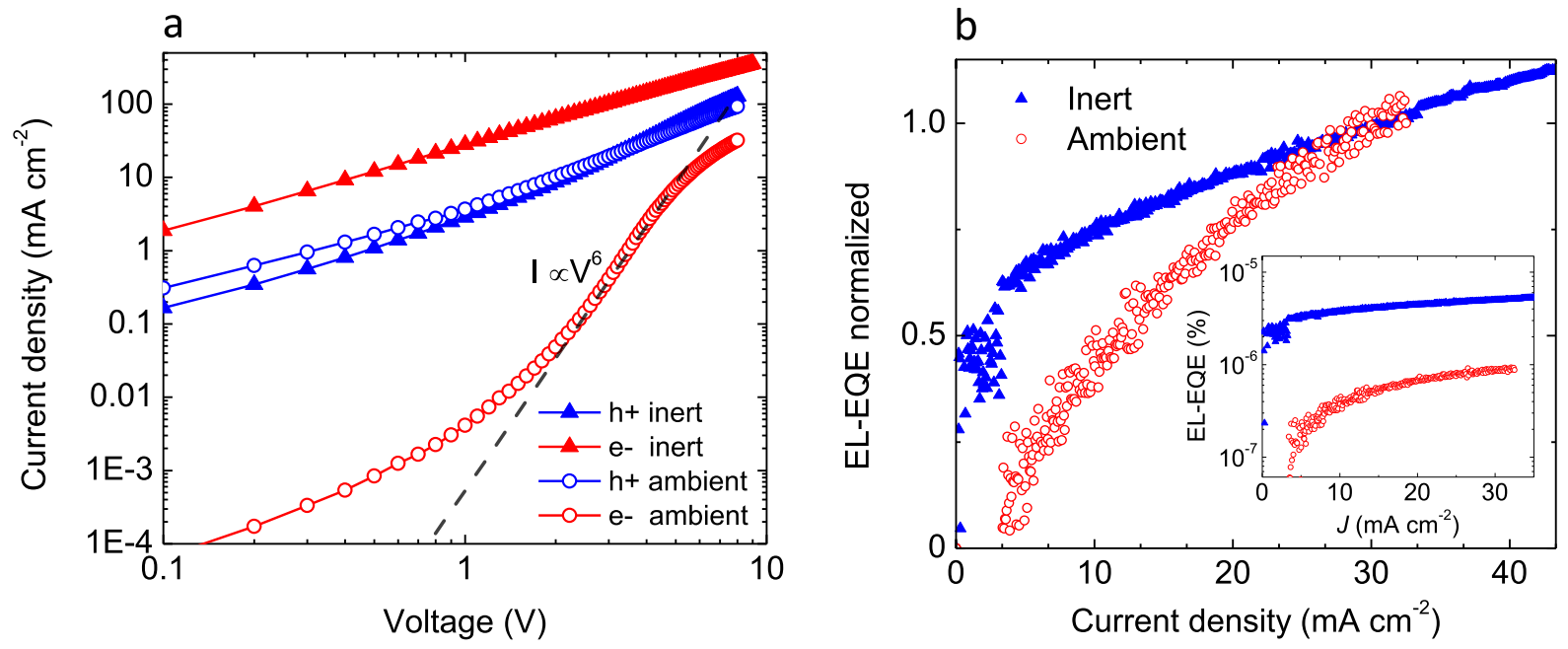

Fig. 5 Carrier transport a SCLC plot of laminated hole-only (blue) and electron-only (red) devices processed inert and exposed to ambient for 2 (filled triangles) and 45 (open circles) minutes. b EL-EQE vs. injected current-density for a laminated TQ1:PC ${ }_{71} B M$ device processed inert (blue triangles) and exposed to ambient for $45 \mathrm{~min}$ (red circles)

that voltage-dependent charge transfer (CT) electroluminescence quantum efficiency (EL-EQE) measurements can be used to identify the presence of non-radiative trap-assisted recombination. ${ }^{24}$ The CT EL-EQE is bias-independent in the case of bimolecular recombination and is bias-dependent for trapassisted recombination. ${ }^{24}$ Figure $5 \mathrm{~b}$ shows the measured EL-EQE for $\mathrm{TQ1}: \mathrm{PC} \mathrm{C}_{71} \mathrm{BM}$ devices processed under inert conditions and in ambient. We find that the ambient-processed device has an order of magnitude lower CT EL-EQE and a stronger dependence of CT EL-EQE on injected current density (Fig. 5b). Both observations are indicative of non-radiative trap-assisted recombination in the ambient-processed device. On basis of SCLC and EQE-EL measurements we conclude that exposure to ambient during lamination drastically deteriorates electron transport due to a strong increase of the electron trap density, giving rise to an increased trap-assisted recombination.

The significantly deteriorated electron transport at the anode may cause a pile up of electrons at the anode, i.e., space-charge. EL-EQE shows that trapped carriers recombine, but we also probe the presence of space-charge effects for photo-generated carriers by light-intensity-dependent photocurrent measurements (Fig. 6a). From $J_{\mathrm{SC}} \propto \mathrm{I}^{\mathrm{a}}$, we fit $a$, where $a=1$ is an indication of weak bimolecular recombination, whereas $a=0.76$ originates due to space-charge effects. ${ }^{25}$ We see no major differences in the slope as $a \sim 1$ for all devices, which means that space-charge effects do not play a significant role. This is in contrast to previous studies reporting asymmetric photocurrent extraction, which was attributed to space-charge effects. ${ }^{26-28}$ Thus, on basis of light-intensity dependent $J_{\text {SC }}$ measurements we rule out space-charge effects as the origin of asymmetric photocurrent collection.

Due to the presence of electron traps in ambient exposed devices, the recombination mechanisms are expected to be different in AL1 and AL2, i.e., closer to the anode and the cathode electrodes, respectively. To investigate the difference in the recombination mechanisms at the two electrodes we have carried out light-intensity-dependent $V_{\mathrm{OC}}$ measurements (Fig. 6b). The slope $(S)$ of the $V_{O C}$ vs. illumination intensity can be used to determine the dominant recombination mechanism: $S=1$ for bimolecular recombination and $S>1$ is typically assigned to trapassisted recombination. ${ }^{29}$ The increased slope for ambientexposed devices is an indication of an increasing importance of trap-assisted recombination and we find a higher slope upon illumination from the anode, compared to the cathode. We have further confirmed that recombination is different depending on what side is illuminated by a recently developed techniqueintermodulation photocurrent (IMP), ${ }^{30}$ see the Supporting Information Supplementary Fig. SI7. From the light-intensitydependent $V_{\mathrm{OC}}$ and IMP measurements we conclude that the amount of trap-assisted recombination increases upon ambient exposure and that it is more pronounced when illuminating from the anode side, as expected.

Our investigations have thus shown that the main cause of the asymmetric photocurrent collection are electron traps in the active layer, hampering electron transport through the device and causing trap-assisted recombination. However, we cannot explain the chemical nature of these traps. We speculate that the ambient-induced asymmetry could be caused by water molecules trapped in voids in the AL. Water molecules have been recently reported to induce traps in conjugated polymers for field-effect transistors, ${ }^{31}$ while oxidation of PCBM is known to also induce traps. $^{32} \mathrm{~A}$ third possibility is that ambient exposure leads to a degradation of the lamination interface, where a region of electron traps is formed, blocking the extraction of electrons generated in AL1. We continue to investigate the degradation mechanisms leading to the increased electron trap density and we are currently searching for spectroscopic signatures of the presence of electron traps in the active layer.

To confirm the generality of our roll lamination method using the active layers as the adhesive we have also used a number of different donor:acceptor combinations. In all cases we have obtained good mechanical and electrical contact, albeit none of the $\mathrm{BHJ}$ systems have been optimized with respect to the PCE (Supplementary Fig. SI8 and Supplementary Table S I1).

The lamination method using $\mathrm{AL}$ as the adhesive opens up additional possibilities to tune the donor/acceptor stoichiometry of AL1 and AI2. We have found that using a pure PCBM layer as $\mathrm{AL} 2$ and a $\mathrm{BHJ}$ as $\mathrm{AL} 1$, the $\mathrm{FF}$ can be enhanced (FF $~ 0.40$ vs. FF $0.55)$. We have attained $\mathrm{PCE}=2.6 \%$ with $\mathrm{TQ1}: \mathrm{PC} \mathrm{C}_{71} \mathrm{BM}$ and $\mathrm{PCE}=$ $3.5 \%$ with the commercial printing ink $\mathrm{PI} 4$ in semitransparent devices using this approach. The corresponding JV-curves are shown in Fig. 7. We have also added a paper reflector to the nonilluminated side of the semitransparent laminated solar cell ${ }^{11}$ and reach $\mathrm{PCE}=3.4 \%$ for $\mathrm{TQ} 1: \mathrm{PC71BM}$ and $\mathrm{PCE}=4 \%$ for $\mathrm{PI} 4$. This is the expected performance of $\mathrm{PI} 4$ in semitransparent PEDOT(PSS) devices and serves as a confirmation that our lamination method can yield as high performance as the sequentially cast devices.

We think that our lamination method could simplify the processing of ternary and quaternary blends as it may give a 

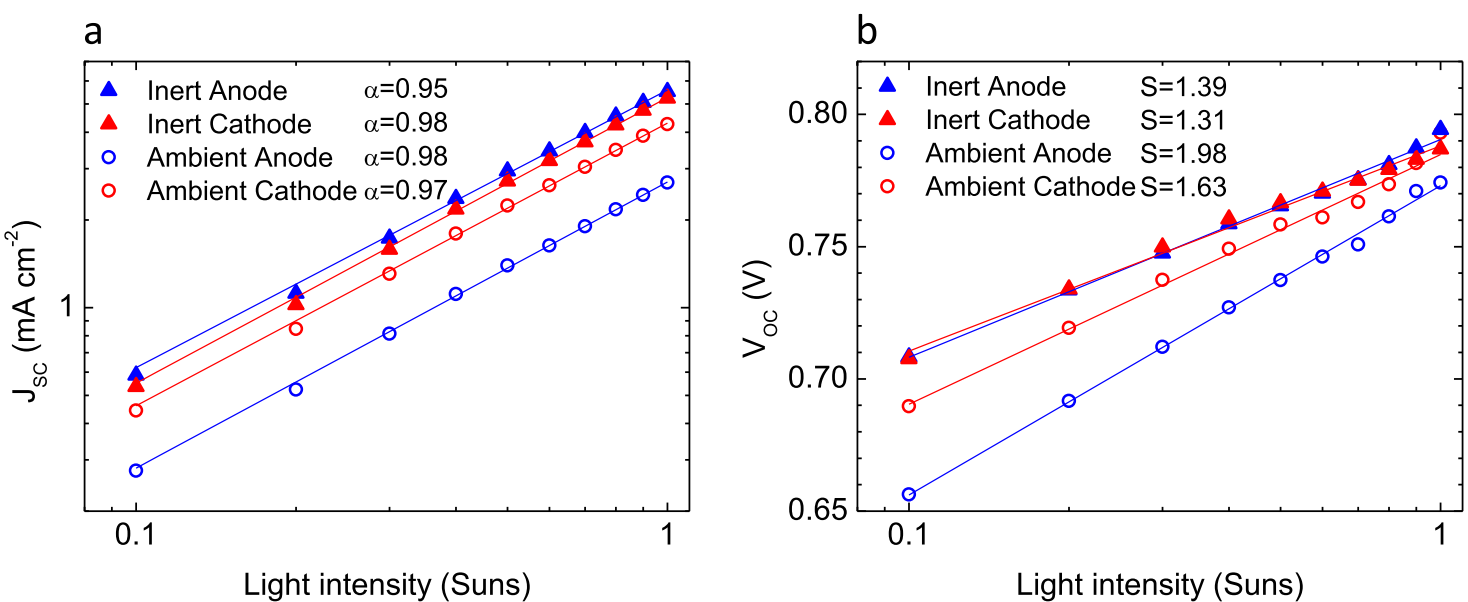

Fig. 6 Light intensity dependence a Short-circuit current and b open-circuit voltage as a function of light intensity for ambient (open circles) and inert devices (filled triangles), for devices illuminated through the cathode (red) and through the anode (blue) side. Solid lines are fits to $V_{\mathrm{OC}}=\mathrm{S}^{*} \mathrm{kT} / \mathrm{q}+\mathrm{m}$ and $J_{\mathrm{SC}}=l^{\alpha}$
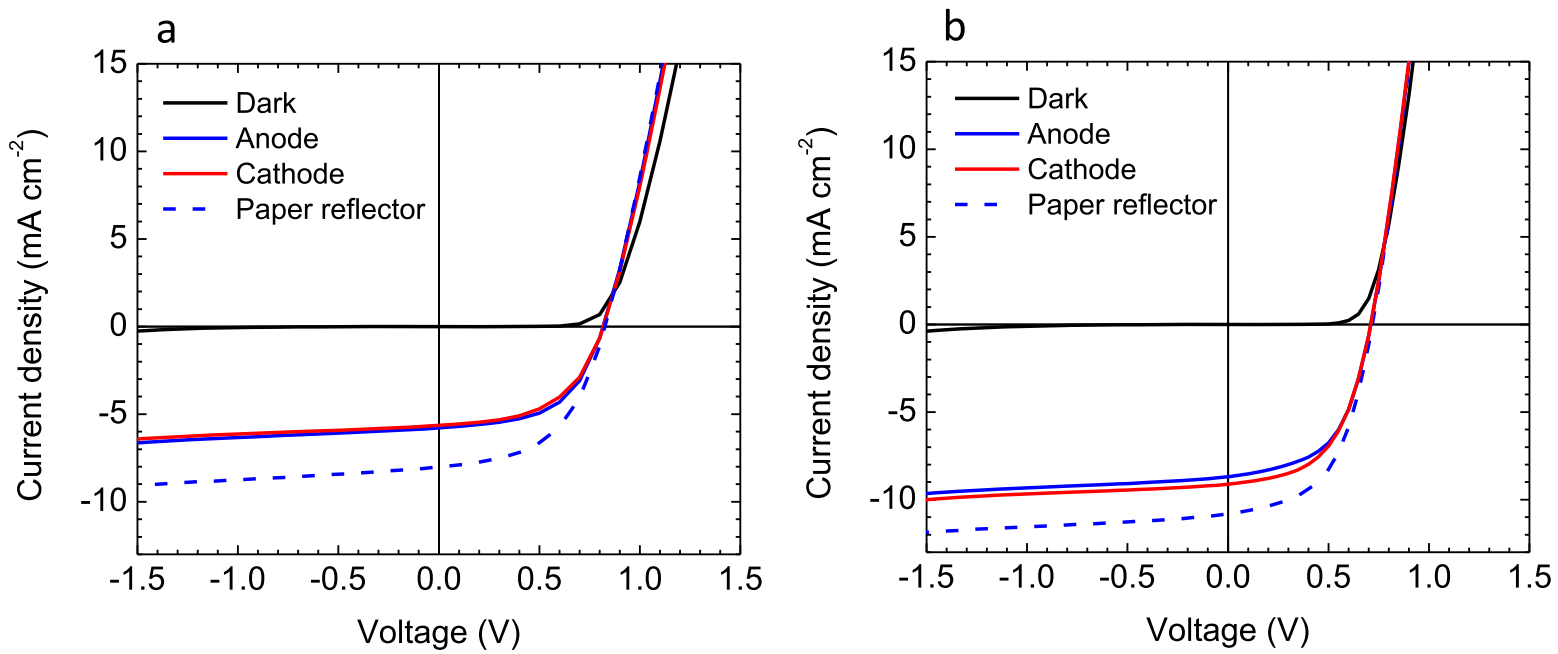

Fig. $7 \quad B H J / P C B M$ lamination JV-curves of $\mathbf{a}$ TQ1:PC 71 BM and $\mathbf{b}$ PI4 laminated A1/A2 BHJ/PCBM semitransparent solar cells illuminated through the anode (solid blue), cathode (solid red) and with the addition of a white paper reflector (dashed blue, illumination from the anode side)

route to control and tune the vertical phase separation. We speculate that in a PCBM/BHJ laminate the fullerenes migrate across the lamination interface during the hot lamination (and possibly the polymer also) and this leads to a preferential gradient with PCBM enrichments closer to the cathode. Furthermore, using the lamination method the stoichiometry at either electrode can be tuned to optimize for favorable vertical phase separation. Thus, active layer lamination could open up a wide range of possibilities for organic solar cells, if the presence of electron traps is avoided, e.g., by processing in an inert environment.

\section{CONCLUSIONS}

In this work we have presented active layer lamination to construct all-organic solar cells, using the active layer as the adhesive layer. This results in well performing solar cells and solves the shunting problem common to devices fabricated using wet deposition of the top PEDOT(PSS) electrode. However, processing in ambient induces a strong asymmetry in photocurrent extraction. The asymmetry originates from ambientinduced electron traps, limiting electron extraction and increasing trap-assisted recombination. We have shown that degradation can be avoided by limiting exposure to ambient. The use of solutionprocessed PEDOT(PSS) electrodes could thus enable truly low-cost OPV, where low material usage significantly reduces the environmental impact and helps improve the return of invested energy. We have shown that the lamination method is universal as we have laminated a number of different polymer:PCBM systems. The performance of the best of our laminated devices is comparable to those expected from sequential wet coating. We expect active layer lamination to be an important tool in future OPV production and research in the context of, i.e., improved control of vertical phase separation with tunable enrichment of the donor and/or the acceptor materials at either electrode.

\section{METHODS}

Laminated solar cells were constructed by laminating two stacks composed of PET/PEDOT(PSS)/PEl/Active layer to Active layer/PEDOT (PSS)/PET using a roll laminator (GSS DH-650S Graphical Solutions Scandinavia AB) with a roll temperature of $120^{\circ} \mathrm{C}$. The force on a force sensor (FlexiForce A201, Tekscan), pushed between the lamination rollers, was measured to $\sim 30 \mathrm{~N}$. The PEDOT(PSS) PH1000 (Heraeus) with 6 vol\% ethylene glycol (Sigma) and 0.5 vol\% Capstone FS-30 (Dupont) and 5-10 $\mathrm{nm}$ thick PEl cathode modification layer coated from isopropanol $\left(1 \mathrm{gl}^{-1}\right)$ 
was slot-die coated (Solar X3, FOM Technologies) on $125 \mu \mathrm{m}$ heatstabilized PET Melinex ST505 (Tekra). More details on the R2R coating line are found in ref. ${ }^{12}$

Active layers were either spin-coated or slot-die coated using a Mini Roll Coater (FOM Technologies) and coated either in a glove box (<10 ppm $\mathrm{O}_{2}$, $\mathrm{H}_{2} \mathrm{O}$ ) or in ambient. TQ1 was synthesized according to ref. ${ }^{7} \mathrm{PC}_{71} \mathrm{BM}(99 \%)$ was bought from Solenne BV and PI4 ink was bought from infinityPV. For the spin coated devices, $\mathrm{TQ1}: \mathrm{PC}{ }_{71} \mathrm{BM}$ active layers were spin coated at 500 rpm from $25 \mathrm{gl}^{-1}$ orthodichlorobenzene solutions and were in total (after lamination) $150-200 \mathrm{~nm}$ thick. The PCBM layer on the cathode for the devices with PCBM/BHJ lamination interfaces were spin coated at 2000 $\mathrm{rpm}$ from a $10 \mathrm{gl}^{-1}$ chloroform solution. PI4 active layers were spin coated at $450 \mathrm{rpm}$ to an expected thickness of $\sim 300 \mathrm{~nm}$. The PI4 based devices were annealed after lamination and encapsulation at $110^{\circ} \mathrm{C}$ for $5 \mathrm{~min}$ on a hot plate. The reported performance of $\mathrm{PCBM} / \mathrm{BHJ}$ devices are hero devices. The width of the laminated solar cells were typically $2 \mathrm{~mm}$ and they were carefully patterned on the PEDOT(PSS) anode with a scalpel in to $\sim 5 \mathrm{~mm}$ wide cells prior to lamination. Photographs of laminated devices are shown in Supplementary Fig. SI8. All samples were encapsulated with glass lids and UV-curing adhesive (Norland NOA73) after lamination. Silver paint (Agar AGG302) was added to the PEDOT(PSS) to reduce the contact resistance to the contacts.

Sequentially cast solar cells were constructed on pre-clean glass substrate. PEDOT electrodes were deposited at a spin-speed of 1500 $\mathrm{rpm}$. The solution was prepared in the same way as that for the laminated devices. The active layer was spin-coated at $1000 \mathrm{rpm}$ from solution based on TQ1:PCBM (1:2.5, weight ratio, ODCB). Solution concentration was 30 $\mathrm{mg} \mathrm{ml}^{-1}$. PEIE (0.05 wt $\%$ in IPA) was deposited at $2000 \mathrm{rpm}$. More details on the sequentially cast devices are found in ref. ${ }^{11}$

PL was measured using a Shamrock SR 303i spectrograph coupled to a Newton EMCCD silicon detector. The samples were excited using a $532 \mathrm{~nm}$ laser diode (CW 532-005, Roithner Lasertechnik).

$J-V$ curves, $V_{\mathrm{OC}}$ and $J_{\mathrm{SC}}$ vs. light intensity were recorded with a Keithley 2400 Source Meter under AM 1.5 G illumination with a solar simulator (LSH7320 LED Solar Simulator, Newport). Illumination intensity was controlled using neutral density filters and the preset intensity setting from 0.1 to 1.0 suns. $V_{\mathrm{OC}}$ and $J_{\mathrm{SC}}$ vs. light intensity measurements were confirmed on two different samples, single carrier device degradation after ambient exposure on at least four different samples and solar cell degradation upon ambient exposure on at least twenty samples. To confirm that no cross talk occurred between cells the resistance between cells was controlled with a multimeter and the neighboring cells were also masked.

EQE was measured using a QE-R (Enlitech). Photocurrent imaging (LBIC) was measured by scanning a red laser diode with $635 \mathrm{~nm}$ emission wavelength (LDM635/5LTM from Roithner Lasertechnik Gmbh) over the solar cell surface. The spot size was $\sim 100 \mu \mathrm{m}$ and the diode was modulated at $3.5 \mathrm{kHz}$ using a multifrequency lock-in amplifier (IMP 2-32 from Intermodulation Products $\mathrm{AB}$ ). The photocurrent response was passed through a pre-amplifier (SR570 from Stanford Research Systems) before being recorded by the lock-in amplifier. To scan the solar cell surface a XY micrometer precision translation table (Nanomotion II, Melles Girot) was controlled and syncronized with the software IMP Suite (Intermodulation Products $A B$ ).

UV-Vis was measured with a Lambda 950 UV-Vis (Perkin Elmer). TMM calculations were performed using an in house Matlab script with optical constants from the local library of optical constants, except for PEI. The optical constants of PEI spin-coated on a silicon substrate was determined using spectroscopic ellipsometry with an RC2 ellipsometer from J.A. Woollam Co., Inc. (USA) and the software CompleteEASE (J.A. Woollam Co., Inc.). Modeling was performed with B-splines.

IMP was measured with two $635 \mathrm{~nm}$ laser diodes (LDM635/5LTM from Roithner Lasertechnik Gmbh) modulated at 3.5 (vertical incidence) and 6 $\mathrm{kHz}\left(20^{\circ}\right.$ incidence angle) using an intermodulation lock-in amplifier (IMP 2-32 from Intermodulation Products $A B$ ). The photocurrent response of the solar cell was pre-amplified (SR570 from Stanford Research Systems) before measured with the lock-in amplifier. The software IMP Suite (Intermodulation Products $A B$ ) was used to control the lock-in amplifier. To confirm overlap between the both illumination spots the 2 nd order IMP signal was monitored live while one laser was carefully moved using a XY manual micrometer stage until the signal reached a maximum.

EQE EL was measured using a homebuilt system using a large-area calibrated Siphotodiode from Oriel.
Data availability

All relevant data are available from authors upon reasonable request.

\section{ACKNOWLEDGEMENTS}

The work was funded by the Swedish Energy Agency, the Swedish Science Council and the Knut and Alice Wallenberg foundation (KAW), through generous instrument grants and a Wallenberg Scholar grant to O.I. We acknowledge Anders Elfwing at Linköping University for helpful discussions. We thank Wolfgang Tress for drift diffusion simulations.

\section{AUTHOR CONTRIBUTIONS}

J.B. and T.Ö. contributed equally to this work. J.B. planned the experiments, measured photocurrent imagining, side-dependent $\mathrm{PL}, \mathrm{IMP}$, made the TMM calculations, analyzed the data, and wrote the manuscript. T.Ö. made all laminated devices, measured JV, UV-Vis and SCLC, contributed to the analysis and revised the manuscript. A.M. measured EL-EQE, contributed to the analysis of the data and revised the manuscript. L.E.A. measured EQE and $V_{\mathrm{OC}}$ and $J_{\mathrm{SC}}$ vs. illumination intensity and revised the manuscript. Z.T. made the sequentially cast solar cells, contributed to the method development and revised the manuscript. W.C. contributed to the method development and revised the manuscript. Z.M. measured PL during lamination and revised the manuscript. D.G. and M.A. contributed with the upscaled synthesis of TQ1 and P3TI polymers used and revised the manuscript. M.K. contributed to the data analysis and discussions and revised the manuscript. O.I. initiated the project and contributed to analysis and discussions and writing and revising the manuscript.

\section{ADDITIONAL INFORMATION}

Supplementary information accompanies the paper on the npj Flexible Electronics website (https://doi.org/10.1038/s41528-017-0017-6).

Competing interests: The authors declare that they have no competing financial interests.

Publisher's note: Springer Nature remains neutral with regard to jurisdictional claims in published maps and institutional affiliations.

\section{REFERENCES}

1. Espinosa, N., Hösel, M., Angmo, D. \& Krebs, F. C. Solar cells with one-day energy payback for the factories of the future. Energy Environ. Sci. 5, 5117-5132 (2012).

2. Krebs, F. C. Fabrication and processing of polymer solar cells: a review of printing and coating techniques. Sol. Energy Mater. Sol. Cells 93, 394-412 (2009).

3. Li, S. et al. Energy-level modulation of small-molecule electron acceptors to achieve over $12 \%$ efficiency in polymer solar cells. Adv. Mater. 28, 9423-9429 (2016).

4. Espinosa, N. et al. Life cycle assessment of ITO-free flexible polymer solar cells prepared by roll-to-roll coating and printing. Sol. Energy Mater. Sol. Cells 97, 3-13 (2012).

5. Zhang, F. L., Johansson, M., Andersson, M. R., Hummelen, J. C. \& Inganas, O. Polymer photovoltaic cells with conducting polymer anodes. Adv. Mater. 14, 662-665 (2002).

6. Zhou, Y. H. et al. Indium tin oxide-free and metal-free semitransparent organic solar cells. Appl. Phys. Lett. 97, (2010).

7. Kim, Y. H. et al. Highly Conductive PEDOT:PSS Electrode with Optimized Solvent and Thermal Post-Treatment for ITO-Free Organic Solar Cells. Adv. Func. Mater. 21, 1076-1081 (2011).

8. Larsen-Olsen, T. T., Sondergaard, R. R., Norrman, K., Jorgensen, M. \& Krebs, F. C. All printed transparent electrodes through an electrical switching mechanism: a convincing alternative to indium-tin-oxide, silver and vacuum. Energy Environ. Sci. 5, 9467-9471 (2012).

9. Zhou, Y. et al. A universal method to produce low-work function electrodes for organic electronics. Science 336, 327-332 (2012).

10. Galagan, Y. et al. Failure analysis in ITO-free all-solution processed organic solar cells. J. Mater. Chem. A 3, 20567-20578 (2015).

11. Tang, Z. et al. Fully-solution-processed organic solar cells with a highly efficient paper-based light trapping element. J. Mater. Chem. A 3, 24289-24296 (2015).

12. Andersson, L. M. Fully slot-die-coated all-organic solar cells. Energy Technol. 3, 437-442 (2015). 
13. Hösel, M., Søndergaard, R. R., Jørgensen, M. \& Krebs, F. C. Fast inline roll-to-roll printing for indium-tin-oxide-free polymer solar cells using automatic registration. Energy Technol. 1, 102-107 (2013).

14. Sundberg, M., Gustafsson, G. \& Inganäs, O. Rectifying metal-polymer contacts formed by melt processing. Appl. Phys. Lett. 57, 733-734 (1990).

15. Gadisa, A. et al. Transparent polymer cathode for organic photovoltaic devices. Synth. Met. 156, 1102-1107 (2006).

16. Shimada, C. \& Shiratori, S. Viscous conductive glue layer in semitransparent polymer-based solar cells fabricated by a lamination process. ACS Appl. Mater. Interfaces 5, 11087-11092 (2013).

17. Spyropoulos, G. D. et al. Organic and perovskite solar modules innovated by adhesive top electrode and depth-resolved laser patterning. Energy Environ. Sci. 9, 2302-2313 (2016)

18. Kaduwal, D., Zimmermann, B. \& Würfel, U. ITO-free laminated concept for flexible organic solar cells. Sol. Energy Mater. Sol. Cells 120, 449-453 (2014).

19. Huang, J., Li, G. \& Yang, Y. A semi-transparent plastic solar cell fabricated by a lamination process. Adv. Mater. 20, 415-419 (2008).

20. Granström, M. et al. Laminated fabrication of polymeric photovoltaic diodes. Nature 395, 257-260 (1998).

21. Kim, J. B. et al. Modular construction of P3HT/PCBM planar-heterojunction solar cells by lamination allows elucidation of processing-structure-function relationships. Org. Electron. Phys. Mater. Appl. 12, 1963-1972 (2011).

22. Wang, E. et al. An easily synthesized blue polymer for high-performance polymer solar cells. Adv. Mater. 22, 5240-5244 (2010).

23. Pettersson, L. A. A., Roman, L. S. \& Inganäs, O. Modeling photocurrent action spectra of photovoltaic devices based on organic thin films. J. Appl. Phys. 86, 487-496 (1999).

24. Wetzelaer, G.-J. A. H., Kuik, M. \& Blom, P. W. M. Identifying the nature of charge recombination in organic solar cells from charge-transfer state electroluminescence. Adv. Energy Mater. 2, 1232-1237 (2012).

25. Mihailetchi, V. D., Wildeman, J. \& Blom, P. W. M. Space-charge limited photocurrent. Phys. Rev. Lett. 94, 126602 (2005).

26. Büchele, P. et al. Space charge region effects in bidirectional illuminated P3HT: PCBM bulk heterojunction photodetectors. Org. Electron. Phys. Mater. Appl. 22, 29-34 (2015).
27. Tait, J. G. et al. Interfacial depletion regions: beyond the space charge limit in thick bulk heterojunctions. ACS Appl. Mater. Interfaces 8, 2211-2219 (2016).

28. Deledalle, F. et al. Understanding the effect of unintentional doping on transport optimization and analysis in efficient organic bulk-heterojunction solar cells. Phys. Rev. X 5, 011032 (2015).

29. Mandoc, M. M., Kooistra, F. B., Hummelen, J. C., De Boer, B. \& Blom, P. W. M. Effect of traps on the performance of bulk heterojunction organic solar cells. Appl. Phys. Lett. 91, 263505 (2007).

30. Bergqvist, J. et al. New method for lateral mapping of bimolecular recombination in thin-film organic solar cells. Prog. Photovolt. Res. Appl. 24, 1096-1108 (2016).

31. Nikolka, M. et al. High operational and environmental stability of high-mobility conjugated polymer field-effect transistors through the use of molecular additives. Nat. Mater. 16, 356-362 (2017).

32. Reese, M. O. et al. Photoinduced degradation of polymer and polymer-fullerene active layers: experiment and theory. Adv. Funct. Mater. 20, 3476-3483 (2010).

(c) (i)

Open Access This article is licensed under a Creative Commons Attribution 4.0 International License, which permits use, sharing, adaptation, distribution and reproduction in any medium or format, as long as you give appropriate credit to the original author(s) and the source, provide a link to the Creative Commons license, and indicate if changes were made. The images or other third party material in this article are included in the article's Creative Commons license, unless indicated otherwise in a credit line to the material. If material is not included in the article's Creative Commons license and your intended use is not permitted by statutory regulation or exceeds the permitted use, you will need to obtain permission directly from the copyright holder. To view a copy of this license, visit http://creativecommons. org/licenses/by/4.0/.

(c) The Author(s) 2018 